\title{
Biological Invasions in Heterogeneous Environments: The Coupled Map Lattice Framework
}

\author{
L.A.D. Rodrigues, D.C. Mistro* \\ Department of Mathematics, Federal University of Santa Maria, Santa Maria, RS, Brasil.
}

\begin{abstract}
Spatial heterogeneity greatly affects the population spread. Although the theory for biological invasion in heterogeneous spatially continuous habitats have received considerable attention, spatially discrete models have remained outside of the mainstream. In this study, we formulate and analyze a Coupled Map Lattice model for a single species population invading a two dimensional heterogeneous environment. The population growth rate and dispersal coefficient depend on the site quality. We first find an analytical criterium for the spread success in terms of the population growth rate and the dispersal coefficient in unfavorable regions. We then implemented our model for two distinct spatial configurations: periodical stripe-like and randomized environments. The spread rate is computed numerically and it shows a decrease with an increase of the fraction of the hostile sites. However, we observed that invasion success does not depend on the fraction of favorable sites but crucially depends on the connectivity of favorable regions.
\end{abstract}

Keywords and phrases: heterogeneous habitat, coupled map lattices, biological invasions, fragmentation

Mathematics Subject Classification: 92D40

\section{Introduction}

The study of biological invasions is widely recognized as an important issue and has been attracting the attention of the scientific community for decades. Understanding the pattern of spread and estimating the rate at which an invasion takes place is of fundamental importance for designing control and management strategies. Mathematical models have shown to be an important tool as they allow the test of hypothesis and provide insights into the invasion properties and the control strategies performance $[23,30]$.

Since habitat heterogeneity due to natural processes or human disturbances greatly influence the pattern and rate of invasions, much effort has been dedicated to understand its effects on biological invasions. Not only in natural environments but heterogeneity is also observed in well organized habitats such as agriculture plantations for which the study of invasive insects is of great importance. In fact, relevant progress on this subject has been observed since the pioneering work by Shigesada and collaborators [28]. In their seminal paper, they used a reaction-diffusion equations to examine the spatio-temporal dynamics of a single species invading an heterogeneous one dimensional habitat consisting of favorable and unfa-

\footnotetext{
${ }^{*}$ Corresponding author. E-mail: dcmistro@gmail.com
} 
vorable patches alternately arranged. They presented an invasion condition, in terms of the fraction of favorable area, for an introduced species: above a certain threshold of the favorable area the population can invade the habitat by means of a periodic travelling wave. They also calculated the velocity of the travelling periodic wave in terms of the population diffusivity and growth rate. After that, several other aspects have been developed. Kinezaki and collaborators [12] studied the population invasion of a heterogeneous two-dimensional habitat where the favorable and unfavorable sites were alternately arranged in stripes. The existence of travelling periodic waves for periodic habitats and its rate of spread were rigorously analysed $[1,32]$. The effects of the of spatial configuration of the habitat fragmentation on the population invasion has been carried out by Kinezaki and colleagues [14]. Using the framework of reaction-diffusion equations, they analyzed lattice-like corridor environments, island-like and striped habitats and concluded that for each periodically fragmented habitat, the population persistence depends on the fraction of favorable area. Recently, the directional movement towards favorable patches, that is, taxis, has also been taken into consideration in a reaction-diffusion equation model for invasion in heterogeneous periodically habitats and showed to enhance biological invasion in heterogenous habitats [10].

Biological invasion of heterogenous environments through longe-range dispersal was examined by means of Integrodifference equation models [9,31,33].

However, most of the work on biological invasions in heterogenous habitat have considered continuous space models with either continuous time (reaction-diffusion equations) $[14,28]$ or discrete time (Integrodifference equations) [3,9]. Although in the literature there are also works based on spatiallyexplicit simulation models [4,34], to our knowledge, space-discrete system described via Coupled Map Lattices (CML) are out of the mainstream of heterogeneous habitat invasion models. When the individuals present discrete generations (like many insect species that reproduce in a well established period of time), time-discrete models are more adequate than the continuous ones. Furthermore, if the space, at some convenient scale of observation, has a markedly discrete feature such as citrus groves where each tree can be viewed as a patch or systems of agricultural fields, then a description via the CML formalism would be more appropriate.

This approach, which constitutes a powerful tool to analyze spatial and temporal patterns in biological interactions, has been used in the last three decades to study many problems such as host-parasitoid interactions [5,19], dispersal-driven instability [24,25,35], biological invasions $[2,20]$ and critical patchsize $[18]$.

In this paper, we propose a simple CML model for single species invasion process in heterogeneous habitat. In Section 2, we describe the model formulation. Specifically, we consider that the growth and movement rates depend on the habitat quality which is classified as favorable or unfavorable. We consider that movement rate in favorable sites is less than that in the unfavorable ones to reflect the tendency of individuals to stay longer in suitable regions and flee from hostile sites. We further employ a heuristic method to derive an invasion condition depending on the distance between favorable patches. In Section 3 numerical simulations are presented for two distinct habitat configurations: periodically alternated stripes of favorable and unfavorable sites and randomized fragmented habitat. Finally, we reserved Section 4 for the discussion of the results.

\section{Discrete Model for Dispersion in Heterogeneous Habitat}

In this section, we describe the proposed two dimensional CML model in order to study a single species invasion process in a heterogeneous habitat. It could represent, for instance, an herbivorous insect introduced in a heterogeneous plantation or natural environment, which exhibit favorable high plant quality patches and unfavorable (or even inhospitable) low quality sites arranged so that high and low quality patches compose a heterogeneous habitat. We assume that in the time scale of observation, the landscape does not change, that is, there is neither depredation by the population nor growth of the resources. 
In the two dimensional CML model, the habitat is split into patches or sites $\mathbf{x}_{i}$ that are arranged in a grid or lattice $\Omega=\left\{x_{-\infty}, \ldots, x_{0}, \ldots, x_{\infty}\right\} \times\left\{y_{-\infty}, \ldots, y_{0}, \ldots, y_{\infty}\right\}$ and represent real locations where the population is distributed. At each lattice point $\mathbf{x}_{i}=\left(x_{i}, y_{i}\right)$ (with $x_{i}=i h, y_{i}=i h$, where $h$ is the patch size and $i$ is an integer) localized at the center of the patch, we represent the mean population density over the patch [2].

The individuals are supposed to present discrete generations and the system dynamics consists of two distinct stages: a sedentary reaction stage and a stage of dispersion. Population growth occurs in the sedentary reaction stage while in the dispersal stage, individuals can move to neighboring patches or remain in their original patch $[5,24,35]$.

The update rule for the density of individuals in each patch takes into account the two distinct process above mentioned so that, at each time step, two phenomena occur: a) population growth, described by the equation:

$$
N_{\mathbf{x}_{i}, t}^{\prime}=f\left(N_{\mathbf{x}_{i}, t}, \mathbf{x}_{i}\right),
$$

where $f$ takes into account the species life history and dependence on the spatial position and b) spatial movement, in which a fraction of individuals leaves their home position and colonize the neighbouring sites while the remainder fraction stay in its original position. The dispersal stage is described by the equation:

$$
N_{\mathbf{x}_{i}, t+1}=\sum_{\mathbf{x}_{j} \in V_{\mathbf{x}_{i}}} k\left(\mathbf{x}_{i}, \mathbf{x}_{j}\right) N_{\mathbf{x}_{j}, t}^{\prime}
$$

where $N_{\mathbf{x}_{i}, t}$ is the population size in site $\mathbf{x}_{i}$ at time step $t, N_{\mathbf{x}_{i}, t}^{\prime}$ is the population size before dispersal and after reaction. We assume that migration occurs only between the closest neighboring sites (that is, individuals are not able to develop long-jump dispersal). Hence for $V_{\mathbf{x}_{i}}$ we choose the von Neumann neighbourhood including the central patch so that, the number of neighbouring sites is $\left|V_{\mathbf{x}_{i}}\right|=5 . k\left(\mathbf{x}_{i}, \mathbf{x}_{j}\right)$ is a discrete dispersal kernel, it indicates the fraction of individuals that migrate from site $\mathbf{x}_{j}=\left(x_{j}, y_{j}\right)$ to site $\mathbf{x}_{i}$ during the dispersal process. It depends on the species behaviour and individuals response to the environment and will be described below.

Equation (2.1) and (2.2) can be rewritten in the more concise form

$$
N_{\mathbf{x}_{i}, t+1}=\sum_{\mathbf{x}_{j} \in V_{\mathbf{x}_{i}}} k\left(\mathbf{x}_{i}, \mathbf{x}_{j}\right) f\left(N_{\mathbf{x}_{j}, t}, \mathbf{x}_{j}\right) .
$$

We incorporate the heterogeneity of the habitat by assuming that the growth rate and the fraction of individuals that leave its position are not constant but vary depending on the environment quality.

As for the reaction stage, we will assume the non-dimensional Ricker function [9] as a paradigm of growth:

$$
f\left(N_{\mathbf{x}_{i}}, \mathbf{x}_{i}\right)=N_{\mathbf{x}_{i}} e^{r_{\mathbf{x}_{i}}-N_{\mathbf{x}_{i}}}
$$

where $e^{r_{\mathbf{x}_{i}}}$, the intrinsic growth rate, depends explicitly on $\mathbf{x}_{i}$ as a consequence of the habitat heterogeneity. So, we define

$$
r_{\mathbf{x}_{i}}=\left\{\begin{array}{l}
r_{1}, \quad \text { if } \mathbf{x}_{i} \text { is a favorable site } \\
r_{2}, \text { if } \mathbf{x}_{i} \text { is an unfavorable site, }
\end{array}\right.
$$

where $r_{1}>r_{2}$ indicates that the population growth in favorable is higher than in unfavorable patches.

The redistribution kernel, in its turn, depends not only on the distance between sites but depends explicitly on $\mathbf{x}_{j}$,

$$
k\left(\mathbf{x}_{i}, \mathbf{x}_{j}\right)=k\left(\delta, \mathbf{x}_{j}\right)=\left\{\begin{array}{cc}
\left(1-\mu_{\mathbf{x}_{j}}\right) & \text { if } \quad \delta=0, \\
\frac{\mu_{\mathbf{x}_{j}}}{4} & \text { otherwise }
\end{array}\right.
$$


where $\delta=\left|x_{i}-x_{j}\right|+\left|y_{i}-y_{j}\right|$ and the dispersal coefficient $\mu_{\mathbf{x}_{j}}$, is defined by the piecewise function:

$$
\mu_{\mathbf{x}_{j}}=\left\{\begin{array}{l}
\mu_{1}, \quad \text { if } \mathbf{x}_{j} \text { is a favorable site } \\
\mu_{2}, \text { if } \mathbf{x}_{j} \text { is an unfavorable site }
\end{array}\right.
$$

where $0 \leq \mu_{1,2} \leq 1$.

In this work we consider that individuals have a limited sensorial perception so that they can only identify their current location as favorable or not but cannot qualify their neighbouring sites in order to decide where they should move $[8,26]$. Furthermore, individuals of many species disperse with less intensity in favorable regions and, in this way, stay longer in high quality positions. In an attempt to explain the tendency of the individuals to remain longer in favorable regions, several model formulations have been proposed in the specialized literature $[6,7,16]$. We describe this tendency by considering the fraction of individuals that leaves its position to be small on favorable sites and great in the unfavorable sites, so that $\mu_{2}>\mu_{1}$.

\subsection{Species Introduction and Establishment}

It is well recognized that not every introduction results in successful invasion [30]. Biological invasions consist of three distinctly different stages such as (i) alien species introduction, (ii) establishment and (iii) spatial spread. While the conditions for a successful spread are well understood in space-continuous systems $[3,9,12,27,28]$, to our knowledge, no work has been done to reveal the corresponding condition for alien species spread in heterogeneous space-discrete systems. This problem is addressed in this section.

The linear conjecture [21], widely used in continuous space models [3,32,33] for obtaining an approximation for the spread rate, will be used here for achieving a criterium for the successful population spread. We suppose that on the leading edge, the population density is low and then the equation (2.4) can be approximated by the linearized equation

$$
N_{\mathbf{x}_{i}, t+1}=\sum_{\mathbf{x}_{j} \in V_{\mathbf{x}_{i}}} k\left(\mathbf{x}_{i}, \mathbf{x}_{j}\right) R\left(\mathbf{x}_{j}\right) N_{\mathbf{x}_{j}, t}
$$

where $R\left(\mathbf{x}_{j}\right)=\frac{\partial f}{\partial N}\left(0, \mathbf{x}_{j}\right)$ is the linearized growth rate. For the growth function $(2.4), R\left(\mathbf{x}_{j}\right)=R_{1}=e^{r_{1}}$ on a favorable patch while $e^{r_{2}}=R_{2}$ corresponds to the linearized growth rate on unfavorable patches. Notice that $R_{1}>R_{2}$ and $R_{2}<1$ indicates a completely hostile patch. It is important to mention that for $r_{2}>0$ the population spread is always possible and for this reason, in what follows we consider $r_{2}<0$.

We now consider an island-like environment where the favorable regions consist of squares $L_{1} \times L_{1}$ sites regularly distributed in the environment and separated by $L_{2}$ unfavorable sites in each direction, as illustrated in Figure 1. For simplicity we take $L_{1}=1$ and then suppose that $N_{0}$ individuals are released in a favorable site $\mathbf{x}_{0}=\left(x_{0}, y_{0}\right)$ located at the center of the habitat. We observe the evolution of the population density in the horizontal direction; it will occur the same in all directions. After $t^{*}$ time steps individuals travel $t^{*}$ hostile sites so that, the density in patch $\left(x_{t^{*}}, y_{0}\right)$, is

$$
N_{\left(x_{t^{*}}, y_{0}\right), t^{*}}=\frac{\mu_{1} R_{1} N_{0}}{4}\left(\frac{\mu_{2} R_{2}}{4}\right)^{t^{*}-1} .
$$

With the purpose of avoiding undesirable artificial results, such as population growth from densities of the order of $10^{-30}$, below a critical value $N_{c} \approx 0$ we will set the population density as zero. Hence, in order to spread, the population must not vanish before it reaches a favorable patch, that is, it must exceed the critical value $N_{c}$. This hypothesis gives us the maximum number of unfavorable sites $t^{*}$, henceforth denoted by $L_{2}^{*}$, for the population successful spread,

$$
L_{2}^{*}=\left\lfloor\log _{\frac{\mu_{2} R_{2}}{4}} \frac{\mu_{2} R_{2} N_{c}}{\mu_{1} R_{1} N_{0}}\right\rfloor,
$$




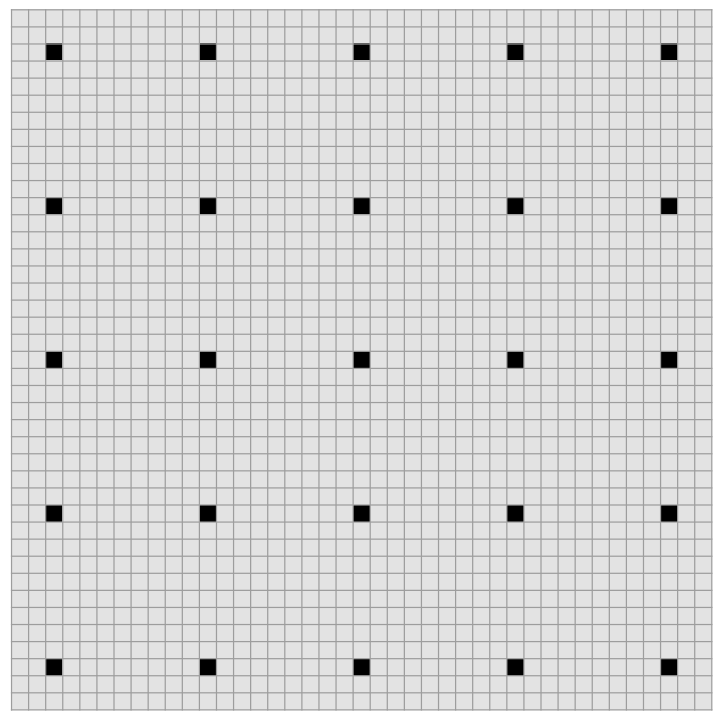

Figure 1. Portion of the island-like habitat for $L_{1}=1$ and $L_{2}=8$. Black color represents favorable habitat while gray corresponds to hostile sites.

where $\lfloor x\rfloor$ represents the largest integer less than $x$.

If the closest favorable patch is located more than $L_{2}^{*}$ patches away from the original one, individuals are not able to cross the hostile region, that is, they die out before reaching the another favorable site. In this case, the population cannot spread and remain confined to the original site. On the other hand, if favorable sites are located less than $L_{2}^{*}$ from one another, individuals can reach other favorable sites and the population spread is guaranteed.

The piecewise constant curve in Figure 2 illustrates $L_{2}^{*}$, given by (2.10), as a function of the growth rate in unfavorable sites $r_{2}$, for $r_{1}=1.8, \mu_{1}=0.1, \mu_{2}=0.9, N_{0}=1$ and $N_{c}=10^{-10}$ fixed. For $L_{2}$ taken in the gray region, the population spread takes place. The dots indicate the results obtained by simulations with the whole nonlinear model (2.3) with favorable sites arranged as in the island-like scenario described before and $N_{0}=1$ individuals initially released in the favorable central site. For each value of $r_{2}$, we increased $L_{2}$ from $L_{2}=1$ and found its greatest value for which the spread succeeds, that is, $L_{2}^{*}$. We can observe in Fig. 2 that if $L_{2}$ is small enough, the invasion always occurs, as it was observed in a similar integrodifference model by [9]. However, differently from what was obtained by Kawasaki and Shigesada [9], we observed that for negative close to zero values of $r_{2}$, there will be a sufficiently large value of $L_{2}$ for which the spread will fail.

The success of the invasion does not depend on $L_{1}$, the width of the favorable region where the individuals are introduced, as it will be confirmed in the next section through numerical simulations for the stripe-like habitat. The invasion accomplishment depends on how hostile and how large the unfavorable region is. We point out that we chose the simplest environment configuration for the criterium achievement, however, we will heuristically use (2.10) for other habitat configuration (stripe-like and randomized environment).

\section{Simulation}

Since we are interested in investigating the dispersal process of an initially introduced population, we considered a sufficiently large domain so that the effects of the boundaries are negligible during the time considered. As initial condition, we assume a small inoculation of individuals in the center of the lattice 


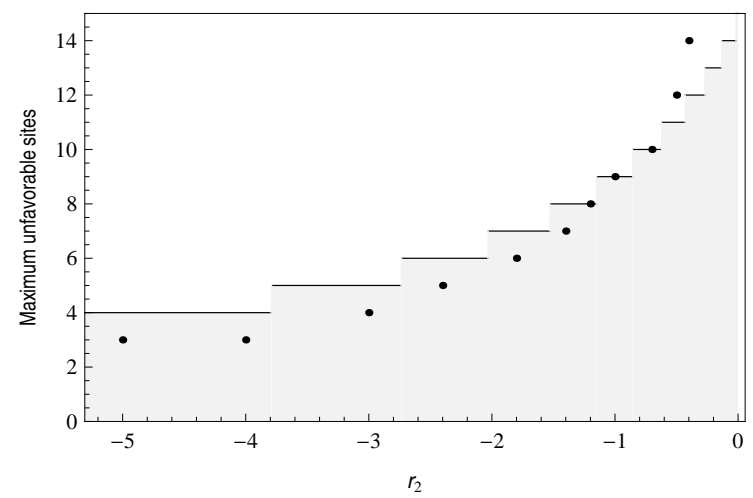

Figure 2. Maximum number of unfavorable sites for the population spread, $L_{2}^{*}$, as a function of the growth rate in the hostile sites $r_{2}$, for $r_{1}=1.8, \mu_{1}=0.1, \mu_{2}=0.9$, $N_{c}=10^{-10}$ and $N_{0}=1$. Dots represent the value of $L_{2}^{*}$ obtained by simulations with the whole nonlinear model (2.3) and island-like environment.

and study Eqs. (2.3) with (2.4) to (2.7) by means of numerical simulations for two different spatial configuration of the resources: striped and randomized environments, which will be described below.

\subsection{Striped Habitat}

In this numerical experiment, we consider the single species invasion in a stripe-like periodic environment where the favorable and unfavorable patches are alternately arranged in the two dimensional grid so that a favorable stripe is located at the center of the habitat, where the individuals are initially released. In this context, parameters (2.5) and (2.7) are defined as:

$$
r_{\mathbf{x}_{i}}=\left\{\begin{array}{l}
r_{1}, \text { if } \quad q L-L_{1} \leq x_{i}<q L \\
r_{2}, \text { if } q L \leq x_{i}<q L+L_{2}, q \in Z,
\end{array}\right.
$$

and

$$
\mu_{\mathbf{x}_{i}}=\left\{\begin{array}{l}
\mu_{1}, \text { if } \quad q L-L_{1} \leq x_{i}<q L \\
\mu_{2}, \text { if } \quad q L \leq x_{i}<q L+L_{2}, q \in Z
\end{array}\right.
$$

where $L_{1}$ and $L_{2}$ represent, respectively, the width of favorable and unfavorable stripes and $L=L_{1}+L_{2}$. In this scenario, the fraction of favorable sites and the fraction of unfavorable sites are, respectively, $\frac{L_{1}}{L_{1}+L_{2}}$ and $\frac{L_{2}}{L_{1}+L_{2}}$. We recall that $r_{1}>r_{2}$, that is, the growth rate in the favorable sites is greater than that in the hostile sites while $\mu_{1}<\mu_{2}$, that is, the motility rate in the unfavorable sites is greater than that in the favorable ones.

We observed that when the distance between two favorable stripes is greater than $L_{2}^{*}$ sites, the invasion fails and the population, initially released in a favorable stripe, colonizes all the stripe extension and remains confined to it. On the other hand, if the distance between favorable stripes is less than $L_{2}^{*}$ patches, the invasion occurs through a propagating front similar to those obtained by Kawasaki and Shigesada [9] for integrodifference equation models. The shape of the front varies with time, so that no superposition of the front in different time-steps can be obtained by shifting. The typical population spatial configuration is illustrated in Figure 3 where the dark (light) gray colors indicate high (low) individuals density. Figure 3(a) corresponds to $L_{2}=2$ while fig. 3(b) depicts the population distribution for $L_{2}=10$, both for $L_{1}=1$ at $t=50$.

The spread rate is different in each direction: it is highest along the stripes (over the favorable ones) and is lowest across the stripes. For estimating the spread rate in the direction perpendicular to the 


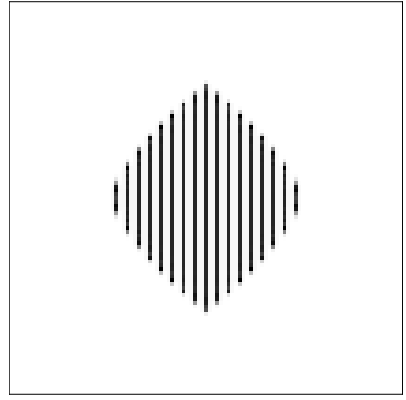

(a)

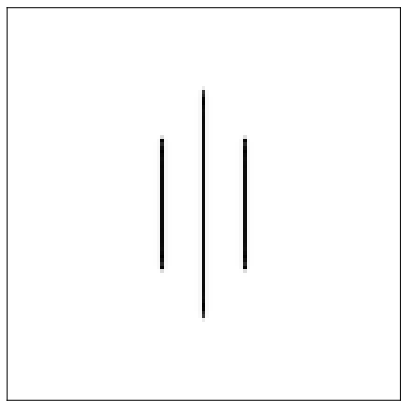

(b)

Figure 3. (a) Spatial distribution of individuals at $t=50$ for the stripe-like environment with $L_{1}=1, L_{2}=2$ and parameters: $r_{1}=1.8, r_{2}=-0.75, \mu_{1}=0.1, \mu_{2}=0.9$. (b) Spatial distribution of individuals at $t=50$ for the stripe-like environment with $L_{1}=1$, $L_{2}=10$ and the same reaction and movement parameters. Dark (light) gray colors indicate high (low) individuals density.

stripes, we adopt the average frontal speed proposed by Morozov and colleagues [22]:

$$
\widetilde{c}=\frac{x_{j, t}^{*}-x_{j, t^{\prime}}^{*}}{t-t^{\prime}}
$$

where $x_{j, t}^{*}$ correspond to the location of the front, in the direction $x_{j}$ and time $t$, where the population density first reaches a detectable small value $N^{*}$. That is,

$$
x_{j, t}^{*}=\max _{x_{j} \in \bar{\Omega}_{t}} x_{j}
$$

and

$$
\bar{\Omega}_{t}=\left\{x_{j} \mid N_{\left(x_{j}, y_{0}\right), t}>N^{*}\right\} .
$$

The time step $t$ in (3.3) must be taken so that the border effects do not affect the results while the time step $t^{\prime}$ must be chosen in order to avoid the impact of the transients on the resulting velocity.

In order to analyse the dependence of the spread rate on the unfavorable stripe width, we fix $L_{1}=1$, $r_{1}=1.8, r_{2}=-0.75, \mu_{1}=0.1$ and $\mu_{2}=0.9$ and vary $L_{2}$. Figure 4 illustrates the average frontal speed in the direction perpendicular to the stripes (which we fixed as the horizontal direction) as a function of $L_{2}$, for $L_{1}=1$ (diamonds), $L_{1}=2$ (squares) and $L_{1}=5$ (circles). We observe that the average spread rate decreases with $L_{2}$ and increases with $L_{1}$. However, population spread is not possible for $L_{2} \geq 11$ regardless how large $L_{1}$ is. Note that criterium (2.10) foresees $L_{2}^{*}=10$ as the maximum unfavorable stripe width for successful spread.

\subsection{Randomized Habitat}

In this second experiment, in order to study the population spread in natural habitats, we use a simple rule for generating a random-like environment. For each site $\mathbf{x}_{i}$, we use the Mathematica's Random function to generate a uniformly distributed pseudorandom real number $r n d_{\mathbf{x}_{i}}$ in the range 0 to 1 . If $r n d_{\mathbf{x}_{i}}<\frac{L_{1}}{L_{1}+L_{2}}$ then the site is qualified as a favorable site, otherwise the site is set as unfavorable. $r_{\mathbf{x}_{i}}$ and $\mu_{\mathbf{x}_{i}}$ are then specified according to (2.5) and (2.7), respectively.

$L_{1}$ and $L_{2}$ have now a different meaning: these parameters are related to the fraction of favorable and hostile sites in the domain, which are the same as before. However, the distance between two different favorable sites is now variable. After the initial release in the central site, individuals must be able to colonize the closest favorable sites and from those, migrate to other favorable patches and so on. We 


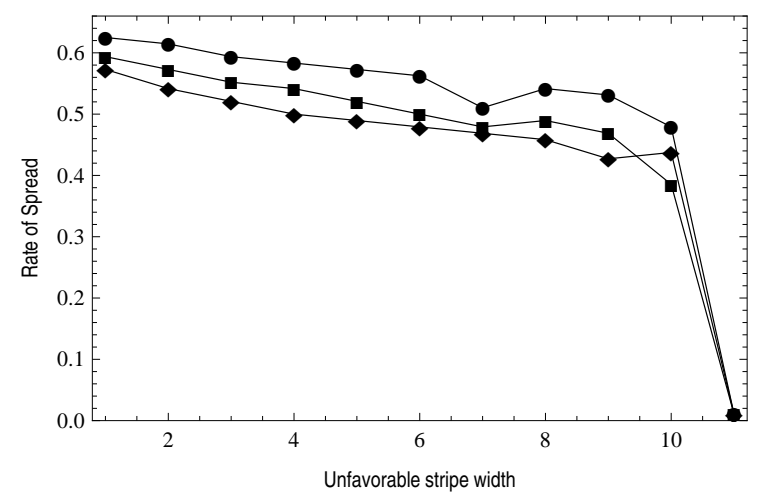

Figure 4. Rate of spread as a function of the unfavorable stripe width $L_{2}$, for $r_{1}=1.8$, $r_{2}=-0.75, \mu_{1}=0.1, \mu_{2}=0.9$ and $L_{1}=1$ (diamonds), $L_{1}=2$ (square) and $L_{1}=5$ (circle).

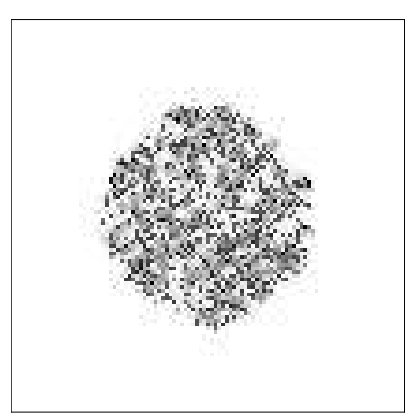

(a)

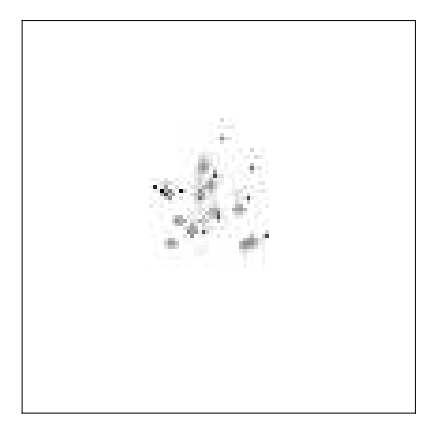

(b)

Figure 5. (a) Spatial distribution of individuals at $t=50$ for the randomized habitat with $L_{1}=1, L_{2}=1$ and parameters: $r_{1}=1.8, r_{2}=-0.75, \mu_{1}=0.1, \mu_{2}=0.9$. (b) Spatial distribution of individuals at $t=50$, for the randomized habitat with $L_{1}=1$, $L_{2}=10$ and the same reaction and movement parameters. Dark (light) gray colors indicate high (low) individuals density.

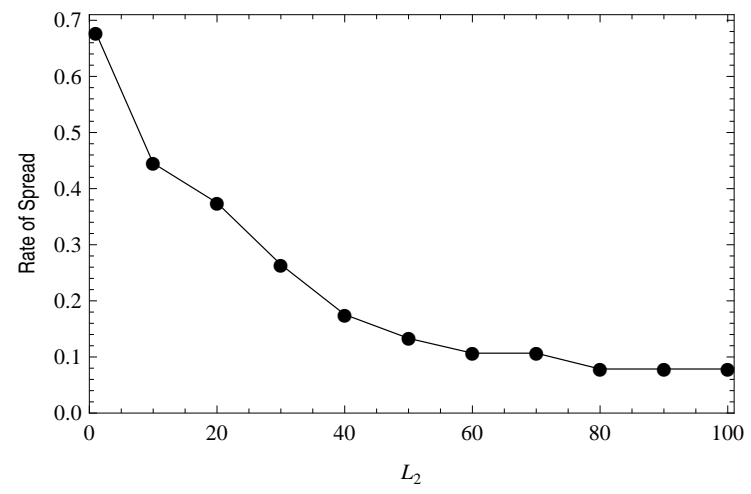

FiguRE 6 . Rate of spread as a function of $L_{2}$, for $L_{1}=1, r_{1}=1.8, r_{2}=-0.75, \mu_{1}=0.1$ and $\mu_{2}=0.9$. 
observe that the spread will not take place if favorable sites are separated by more than $L_{2}^{*}$. On the other hand, if the distance between favorable sites is less than $L_{2}^{*}$ patches then the population spread will occur by means of an irregular front. The population distribution in the colonized region is heterogeneous with different density values in different sites. Figure 5 illustrates the typical configuration of the population distribution for $L_{1}=1$ and $L_{2}=1$ (Fig. 5(a)) and for $L_{1}=1$ and $L_{2}=10$ (Fig. 5(b)).

As for the spread rate, we modify formula (3.3) so that any fixed direction is chosen. We now take

$$
\widetilde{c}=\frac{\mathbf{x}_{i, t}^{*}-\mathbf{x}_{i, t^{\prime}}^{*}}{t-t^{\prime}}
$$

with,

$$
\mathbf{x}_{i, t}^{*}=\max _{\mathbf{x}_{i} \in \overline{\bar{\Omega}}_{t}} \sqrt{\left(x_{i}-x_{0}\right)^{2}+\left(y_{i}-y_{0}\right)^{2}}
$$

where $\mathbf{x}_{0}=\left(x_{0}, y_{0}\right)$ corresponds to the location of the initial individuals release and

$$
\overline{\bar{\Omega}}_{t}=\left\{\mathbf{x}_{i} \mid N_{\mathbf{x}_{i}, t}>N^{*}\right\} .
$$

$\mathbf{x}_{i, t}^{*}$ represents the radius of the colonized region with respect to the central site $\mathbf{x}_{0}=\left(x_{0}, y_{0}\right)$, that is, the location of the front where the population density first reaches a detectable value $N^{*}$.

In order to analyse the effects of habitat fragmentation on the velocity of spread in randomized environments, we fix $L_{1}=1$ and increase $L_{2}$ so that the fraction of favorable sites decreases. We use the Mathematica's SeedRandom function so that the same sequence of pseudorandom numbers is generated. In this way, we keep the same environment scenario and, as we increase $L_{2}$ we increasingly disturb the habitat, such as it occurs in a deforestation processes, for instance.

We consider SeedRandom $=10$ and a $300 \times 300$ grid size while the reaction and movement parameters are taken as before $\left(r_{1}=1.8, r_{2}=-0.75, \mu_{1}=0.1, \mu_{2}=0.9\right)$. The resulting rate of spread as a function of $L_{2}$ is illustrated in Figure 6. As $L_{2}$ increases, the rate of spread decreases but the population fails to invade only for $L_{2}$ sufficiently large. For this particular habitat configuration, invasion still occurs for $L_{2}=100$, which represents approximately $1 \%$ of favorable sites in the habitat. This a very low fraction of favorable habitat compared to the stripe-like scenario, where the invasion fails (with the same reaction and movement parameters) for approximately $31 \%$ of favorable sites $\left(L_{1}=5\right.$ and $\left.L_{2}=11\right)$. In fact, in the stripe-like scenario, invasion will fail for more than $31 \%$ of favorable sites if the width of the unfavorable region is greater than $L_{2}^{*}$.

For a given set of dynamical and movement parameters, the rate of spread and value of $L_{2}$ for the invasion failure in random environments can fluctuate with the value of the SeedRandom and the grid size used in the simulation, since they alter the sequence of pseudorandom numbers and consequently, the habitat configuration and connectivity of favorable sites.

\section{Discussion}

There have been a vast literature focusing on species invasion in continuous heterogenous habitats $[3,9$, $10,12,14,17,27,28]$. These studies focused on the minimum fraction of favorable area for the population persistence and spread, the invasion speed considering random and also directed movement. When the species habitat is distinctly patchy, the spatially discrete models seems to be the most appropriate modelling framework. Although there are several works dealing with simulation models, to our knowledge, there is no attempt to investigate the invasion processes in heterogeneous discrete-space systems.

In this work, we focused on the Coupled Map Lattice (CML) modelling framework to analyze the spatial spread of a single species released in a heterogenous-discrete environment. We first derived an analytical condition for a successful invasion for a regular heterogeneous habitat, which nevertheless, can also be applied to irregular environments. Equipped by this result, we then performed numerical simulations for two spatial configuration: (1) periodic alternating stripes of favorable and unfavorable patches and (2) randomized habitat. 
Contrary to previous findings [14] we observed that it is not the fraction of favorable sites in the environment that determines the invasion success. In discrete-space models, the relevant parameters for a successful invasion of a species, with limited dispersal ability, are the intrinsic growth rate in unfavorable sites and the extent of the hostile region that the individuals must travel before they reach a favorable site. That is, the spatial configuration of favorable and hostile sites which determine the connectivity of the favorable regions is crucial for the population invasion success. Figure 4 shows that in the stripe-like habitat, the population failed crossing $L_{2}=11$ hostile sites for different fractions of favorable sites $\left(\frac{1}{12}\right.$, $\frac{2}{13}$ and $\frac{5}{16}$, for $L_{2}=11$ and $L_{1}=1, L_{1}=2$ and $L_{1}=5$, respectively). Simulations carried out with concentric circular stripes showed results analogous to those obtained with parallel stripes. On the other hand, in the random habitat, the population succeeded invading even when the fraction of favorable sites is very small, such as, $\frac{1}{101}$. Random environments can be easier to invade than stripe-like habitat because of the connectivity of favorable sites. We observed that the rate of spread decreases as $L_{2}$ increases in both habitat scenarios considered.

Our results can be applied either to indicate protecting strategies for species threatened by habitat fragmentation or to suggest measures to control invasive pests or pathogens. Restoration of landscape connectivity can be necessary for native species conservation. On the other hand, barrier zones, made either by spraying toxic substances or by cutting the host plants, can be an alternative to control pests invasions. Agricultural fields interplanted with less attractive cultures can delay an invasive species that develop short range movements and has limited sensorial perception.

We argue that CML constitute a powerful tool for analyzing biological invasions in heterogeneous habitats since, on one hand, its flexibility allows the simulation of a great number of spatial structures and population features, while on the other hand, it can also provide analytical results.

Acknowledgements. LADR was supported by a grant from FAPERGS, process 12/2133-4.

\section{References}

[1] H. Berestycki, F. Hamel, L. Roques. Analysis of the periodically fragmented environment model: II-biological invasions and pulsating travelling fronts. J. Math. Pures Appl. 84 (2005), 1101-1146.

[2] T. de-Camino-Beck, M.A. Lewis. Invasion with stage-structured coupled map lattices: Application to the spread of scentless chamomile. Ecol. Model. 220 (2009), 3394-3403.

[3] C. Dewhirst, F. Lutscher. Dispersal in heterogeneous habitats: thresholds, spatial scales, and approximate rates of spread. Ecology 90 (2009), 1338-1345.

[4] L. Fahrig. Effect of habitat fragmentation on the extinction threshold: a synthesis. Ecol. Appl. 12 (2002), No.2, 346-353.

[5] M.P. Hassell, H.N. Comins, R.M. May. Spatial structure and chaos in insect population dynamics. Nature 353 (1991), $255-258$.

[6] P. Kareiva. Influence of Vegetation Texture on Herbivore Populations: Resource Concentration and Herbivore Moviment in R. F. Denno and M. S. McClure (eds.), Variable Plants and Herbivores in Natural and Managed Systems, 259-289. Academic Press, New York, 1983.

[7] P. Kareiva. Trivial Movement and Foraging by Crop Colonizers in M. Kogan (ed.) Ecological Theory and Integrated Pest Manegement Practice, 59-82. John Wiley \& Sons, New York, 1986.

[8] P. Kareiva, G. Odell. Swarms of predators exhibit "preytaxis" if individuals use area-restricted search. Am. Nat. 130 (1987), No.2, 233-270.

[9] K. Kawasaki, N. Shigesada. An integrodifference model for biological invasions in a periodically fragmented environment. Japan. J. Indust. Appl. Math. 24 (2007), 3-15.

[10] K. Kawasaki, K. Asano, N. Shigesada. Impact of Directed Movement on Invasive Spread in Periodic Patchy Environments. Bull. Math. Biol. 74 (2012), 1448-1467.

[11] T.H. Keitt, M.A. Lewis, R.D. Holt. Allee effects, invasion pinning, and species borders. Am. Nat. 157 (2001), $203-216$.

[12] N. Kinezaki, K. Kawasaki, F. Takasu, N. Shigesada. Modeling biological invasions into periodically fragmented environments. Theor. Popul. Biol. 64 (2003), 291-302.

[13] N. Kinezaki, K. Kawasaki, N. Shigesada. Spatial dynamics of invasion in sinusoidally varying environments. Popul. Ecol. 48 (2006) 263-270.

[14] N. Kinezaki, K. Kawasaki, N. Shigesada. The effect of the spatial configuration of habitat fragmentation on invasive spread. Theor. Popul. Biol. 78 (2010), 298-308.

[15] S. A. Levin. The Problem of Pattern and Scale in Ecology. Ecology 73 (1992), No.6, 1943-1967.

[16] M. A. Lewis, G. Schmitz. Biological Invasion of an Organism with Separate Mobile and Stationary States: Modeling and Analysis. Forma 11 (1996), 1-25. 
[17] F. Lutscher, M. A. Lewis, E. McCauley. The effects of heterogeneity on population persistence and invasion in rivers. Bull. Math. Biol. 68 (2006), No.8, 2129-2160.

[18] V. Méndez, I. Llopis, D. Campos, W. Horsthemke. Extinction and chaotic patterns in map lattices under hostile conditions. Bull. Math. Biol. 72 (2010), 432-443.

[19] D.C. Mistro, L. A. D. Rodrigues, M. C. Varriale. The Role of Spatial Refuges in Coupled Map Lattice Model for Host-Parasitoid Systems. Bull. Math. Biol. 71 (2009), 1934-1953.

[20] D. C. Mistro, L. A. D. Rodrigues, S. Petrovskii.Spatiotemporal complexity of biological invasion in a space- and timediscrete predator-prey system with the strong Allee effect. Ecol. Comp. 9 ( 2012), 16-32.

[21] D. Mollison. Dependence of epidemic and population velocities on basic parameters. Math. Biosci. 107 (1991), $255-287$.

[22] A. Morozov, S. Petrovskii, B.-L. Li. Spatiotemporal complexity of patchy invasion in a predator-prey system with the Allee effect. J. theor. Biol. 238 (2006), 18-35.

[23] A. Okubo, S.A. Levin. Diffusion and ecological problems. (2nd edn.) Springer, Berlin, 2001.

[24] L. A. D. Rodrigues, D. C. Mistro, S. V. Petrovskii.Pattern formation, long-term transients, and the Turing-Hopf bifurcation in a space-and time-discrete predator-prey system. Bull. Math. Biol. 73 (2011), 1812-1840.

[25] L. A. D. Rodrigues, D. C. Mistro, S. V. Petrovskii.Pattern formation in a space- and time-discrete predator-prey system with a strong Allee effect. Theor. Ecol. 5 (2012), 341-362.

[26] L. A. D. Rodrigues, M. C. Varriale, W. A. C. Godoy, D. C. Mistro. Spatiotemporal dynamics of an insect population in response to chemical substances. Ecol. Comp. (2013) accepted.

[27] N. Shigesada, K. Kawasaki. Invasion and the range expansion of species: effects of long-distance dispersal. In: Bullock, J., Kenward, R., Hails, R. (Eds.), Dispersal. Blackwell Science, Oxford, pp. 350-373, 2002.

[28] N. Shigesada, K. Kawasaki, E. Teramoto. Traveling periodic waves in heterogeneous environments. Theor. Popul. Biol. 30 (1986), 143-160.

[29] N. Shigesada, K. Kawasaki, E. Teramoto. The speeds of traveling frontal waves in Heterogeneous environments. In: Teramoto, E., Yamaguti, M. (Eds.), Mathematical Topics in Population Biology, Morphogenesis and Neurosciences. In: Lecture Notes in Biomathematics, vol. 71. Springer, Berlin, pp. 87-97, 1987.

[30] N. Shigesada, K. Kawasaki. Biological Invasions: Theory and Practice. Oxford University Press, 1997

[31] R. W. Van Kirk, M. A. Lewis. Integrodifference models for persistence in fragmented habitats. Bull. Math. Biol. 59 (1997), No.1, 107-137.

[32] H.F. Weinberger. On spreading speeds and traveling waves for growth and migration models in a periodic habitat. J. Math. Biol. 45 (2002), 511-548.

[33] H.F. Weinberger, K. Kawasaki, N. Shigesada. Spreading speeds of spatially periodic integro-difference models for populations with nonmonotone recruitment functions. J. Math. Biol. 57 (2008), 387-411.

[34] K. A. With. The landscape ecology of invasive spread. Cons. Biol. 16 (2002), 1192-1203.

[35] S.M. White, K.A.J. White. Relating coupled map lattices to integro-difference equations: dispersal-driven instabilities in coupled map lattices. J. Theor. Biol. 235 (2005), 463-475. 